\title{
Application of hospital substitutional technologies in rehabilitation of patients after stroke in the late recovery period.
}

\author{
Aleksander Komarov, PhD, MD Clinical Hospital of Administration of President Affairs \\ Ekaterina Silina, PhD, MD Department of Human Pathology of the I.M. Sechenov First Moscow \\ State Medical University.
}

Study Groups

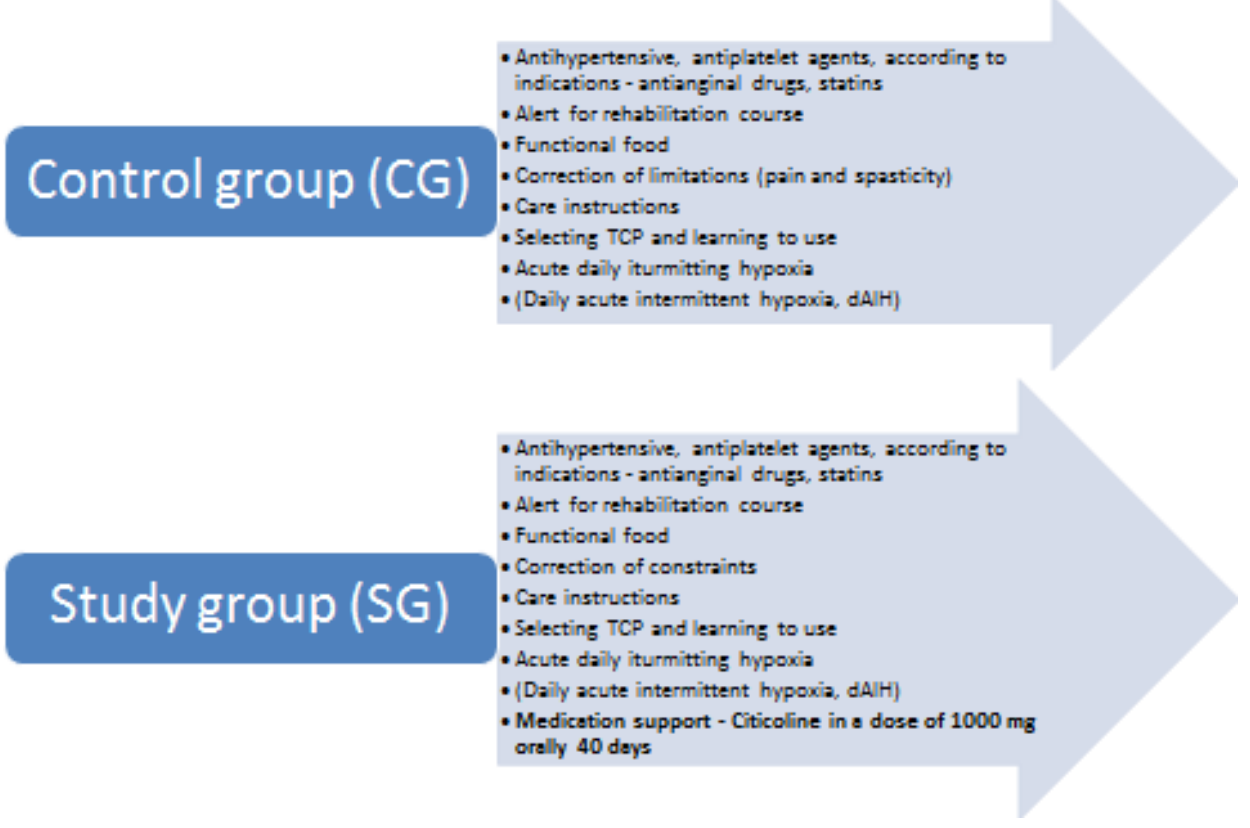

Introduction: Medical-Social rehabilitation - multimodal process. The weakest element is extended outpatient rehabilitation.

Purpose: To study efficiency of comprehensive preconditioning, including treatment with Citicolin of patients in 1.5 years after stroke, at the stage of outpatient rehabilitation.

Objectives: To determine the optimal set of preconditioning techniques for maximum results.

Method: Evaluating of the effeciency of the rehabilitation was carried out on 1 and 30 days on the basis of history, complaints, scale of the NIH, mRs, mobility index Rivermid; universal questionnaire functionality psychometric scales. Statistical analysis was carried out using SPSS 15.0 software using standard parametric and nonparametric statistical significance evaluations. Differences were considered significant at $\mathrm{p}<0,05$.

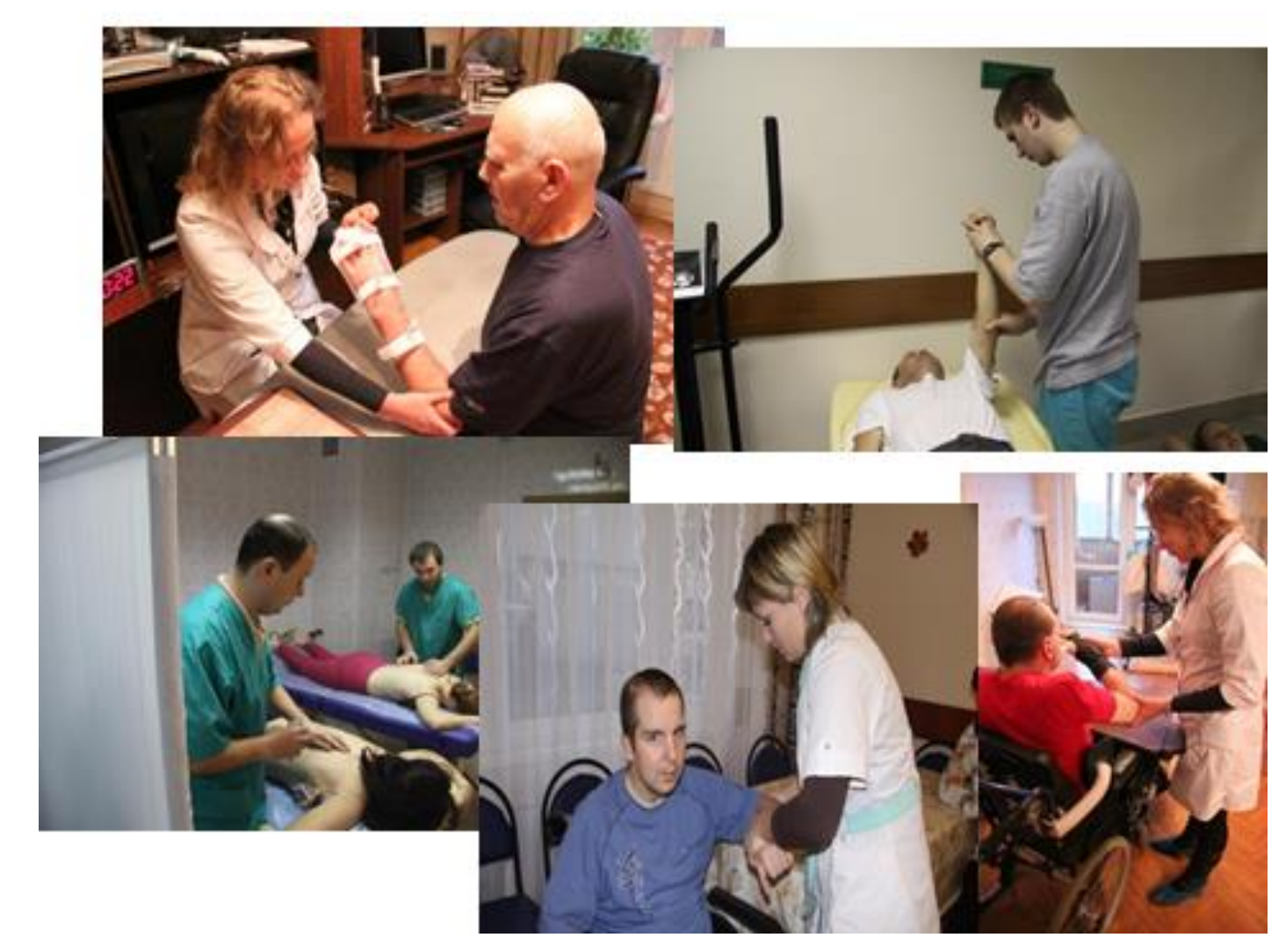

Assessment of clinical efficiency: Evaluating of the effeciency of the rehabilitation was carried out on 1 and 30 days on the basis of history, complaints, somatic and neurological status, scales assessing neurological disorders, the dynamics of social adaptation and patient mobility (scale of the NIH, a modified scale of Rankin, mobility index Rivermid (Rivermead mobility index); universal questionnaire functionality psychometric scales. Statistical analysis was carried out using SPSS 15.0 software using standard parametric and nonparametric statistical significance evaluations. Differences were considered significant at $\mathrm{p}<0,05$.

Results: At the initial examination revealed emotional lability, expressed irritability, increased fatigue and tearfulness in $84.2 \%$ of patients. By 25 days in SG patients, the frequency of these complaints decreased by $42.0 \%$ ( $\mathrm{p}<0.05$ ). During the rehabilitation course, a statistically significant improvement in depression scales (HAM-D), general clinical impression (CGI), anxiety and depression (HADS-21), quality of life assessment (QLS) was observed in patients with SG with various forms of mental disorders ( $\mathrm{P}$ $<0.05)$.
An analysis of the dynamics of the neurologic status registered a more pronounced positive dynamics in the group of patients receiving citicoline. The average regression on the NIH scale in patients who had experienced more than 1.5 years ago stroke for the 30-day course in the CG was 1 point, and in the SG - 2 points, that is, 2 times more. In $52.6 \%$ of the patients enrolled in the study during the outpatient rehabilitation, an improvement in the functional state of the Rivermid Mobility Index was achieved, that is, more than half of the patients who had suffered more than a year and a half ago had stroke and social regimen. Despite the absence of a common inter-group statistically significant difference, the use of citicoline was accompanied by an increase in the proportion of patients with a good functional outcome (0 points on the Rankin scale) in 1.3 times (from $22.2 \%$ to $28.6 \%$ ), and a decrease in the proportion of severe disability (4 points) in 2.9 times (from $13.9 \%$ to $4.8 \%$ ).

\section{Results}

Dynamics of complaints of patients in both groups

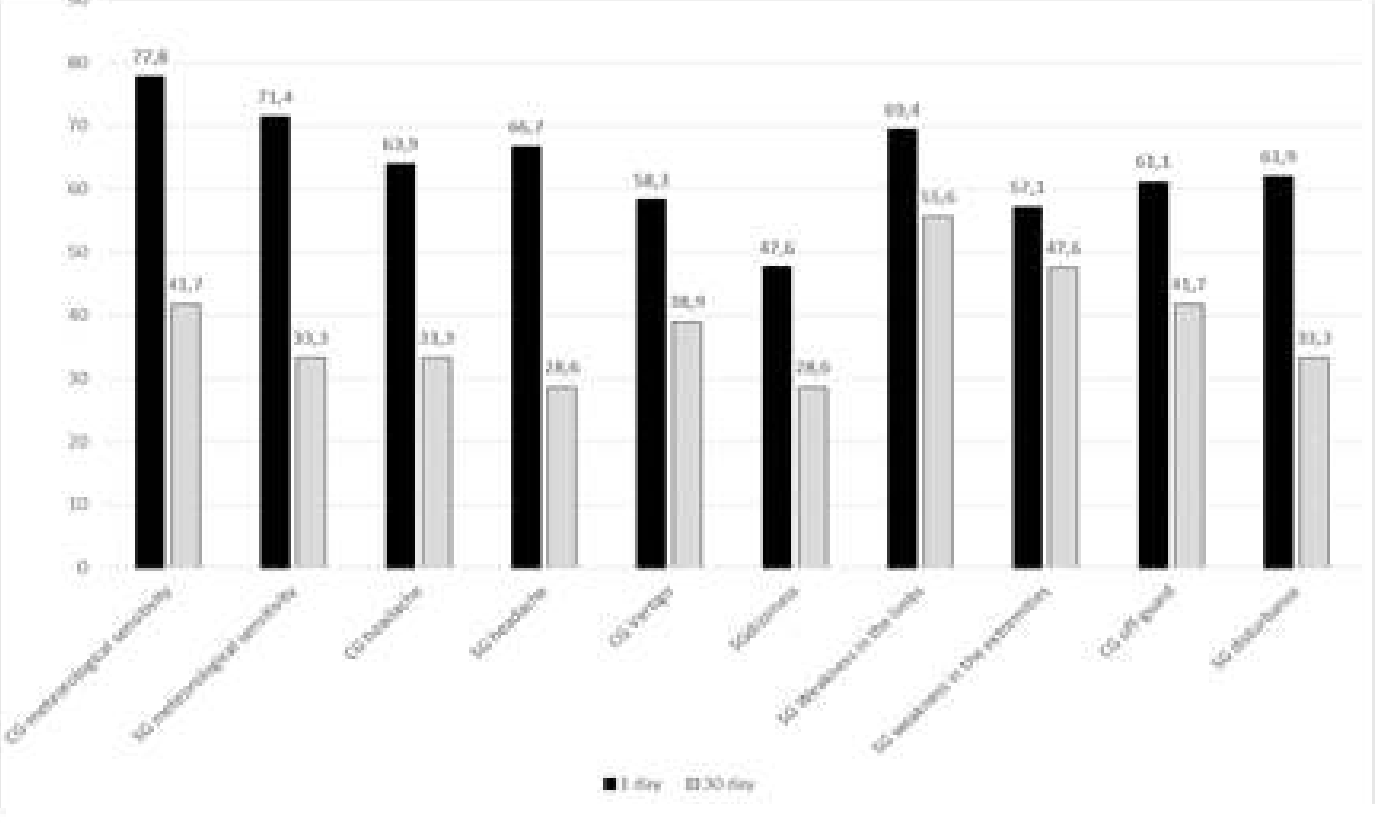

Assessment of functional outcome by Renkin scale
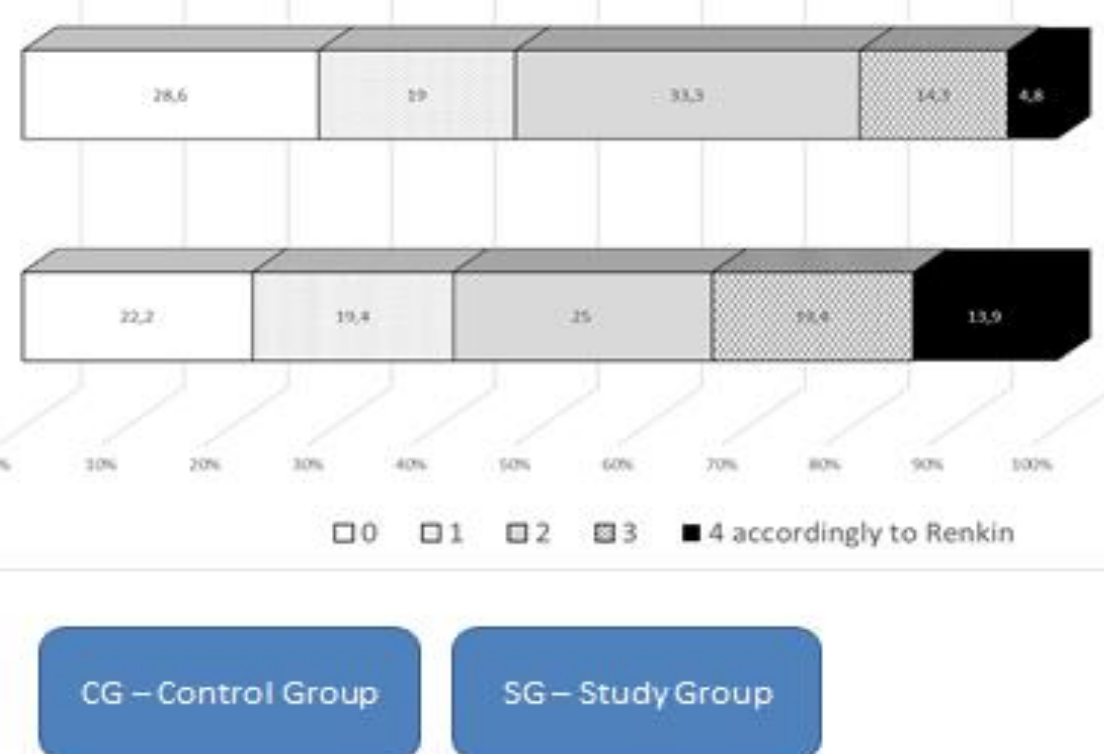

Conclusions: The conducted study demonstrates the effectiveness of complex neurorehabilitation with preconditioning in patients who underwent cerebral stroke more than 1.5 years ago. The use of citicoline within 10 days before the start of rehabilitation activities and 30 days during the rehabilitation process leads to a more pronounced regression of neurological insufficiency and an increase in functional consistency with an increase in the reintegration of disabled people into society. 